\title{
Design and Implementation of a Real-time Traffic Recognition Method based on Video
}

\author{
Xiu Wang ${ }^{1}$, Jianqing $\mathrm{Ye}^{2}$ \\ ${ }^{1)}$ College of Mathematics and Computer Science, Fuzhou University, Fuzhou, Fujian, China (wangx@ fzu.edu.cn) \\ ${ }^{2)}$ China Telecom Fujian Branch, Fuzhou, Fujian, China (yejianqing@ 189.cn)
}

\begin{abstract}
As the increasing of vehicle, the problem of traffic jam is more prominent and the real-time traffic recognition systems come into being. Combine with the characteristic of vehicle distribution in congested roads, a real-time traffic recognition method was designed and implemented by visual space transformation and gridding. This method can recognize different traffic situations effectively and has a good accuracy. It can meet the real-time demands.
\end{abstract}

Keywords - Intelligent Transportation, Real-time Traffic, Traffic Video

\section{基于交通视频的路况分析方法的设计与实现}

\author{
王秀 $^{1}$ 叶剑青 $^{2}$ \\ 1) 福州大学数学与计算机科学学院, 福州, 福建, 中国 \\ 2) 中国电信福建公司, 福州, 福建, 中国
}

摘 要 随着出行车辆数量的增加, 城市交通拥堵问题日益突出, 路况分析系统应运而生。本文结合拥堵道路车辆分布特征, 通 过对道路区域进行视觉空间转换网格化，设计并实现了一种基于交通视频的路况分析方法。通过对结果的分析表明，该方法可有效识 别道路拥堵情况，算法检测准确率高，识别速度快，满足实时性应用的需要。

关键词 智能交通系统，实时路况，交通视频

\section{1. 引言}

随着经济和社会的快速发展, 我国很多城市的市区面 积开始逐渐扩大, 道路建设不断增多, 出行车辆不断增加, 城市交通已经进入一个快速发展的阶段。但是, 很多城市 在发展过程中普遍遇到了城市交通拥堵问题, 解决城区交 通拥堵已成为许多城市迫在眉睫的重要任务 [1]。

智能交通系统 (ITS) 的本质是通过传感技术、信号处 理技术、控制技术等现代信息技术与交通工程的综合应用， 以最大限度地发挥交通基础设施的潜力, 并引导合理的交 通行业 ${ }^{[2]}$ 。通过应用 ITS, 将传统的交通系统改造成一种信 息化、智能化、社会化的新型交通系统。实时路况分析系 统是智能交通系统的一个重要组成部分, 通过实时路况分 析系统, 出行者能够根据自己的意愿了解某条道路或某个 地点的畅通情况, 从而选择最合适的出行行车路线。

基金项目：福州大学科技发展基金（2012-XY-20）
浮动车信息 (FCD) 采集技术是目前国际上 ITS 系统 中采集道路交通信息的主要技术手段，它利用定位技术、 无线通信技术和信息处理技术，实现对道路上行驶车辆的 瞬时速度、位置、路段旅行时间等交通数据的采集。经过 汇总、处理后这些信息生成反映实时道路拥堵情况的交通 信息。虽然 FCD 浮动车数据具有很强的连续性, 可全天候 工作, 提供的交通信息多样化, 但要获得全路网的精确交 通信息就必须要有足够多的装有 GPS 接收设备的车辆驶行 在道路网络中。由于目前浮动车多以安装有 GPS 的营运车 辆为主。在交通高峰期, 运营车辆司机凭借以往经验会在 高峰时段有意避开某些拥堵路段, 导致此路段浮动数据量 较少而与实际路况拥堵情况不相符。而在非高峰时段营运 车辆经常会同时停靠在某些畅通路段（如等客，防止空载 行驶, 节约成本), 导致此类路段停止的浮动车数据量较多, 在地图路况展现上出现严重偏差。此外, 所有的 FCD 浮动 车数据需要经过采集、处理、分析、展示等多个环节, 最 
终在地图上展现往往需要延时 5 至 7 分钟。

本文在对已有的视频处理技术及方法研究的基础上, 提出了一种基于交通视频的实时路况分析方法, 在提高实 时路况准确性与及时性方面起到了较好的效果。该方法可 作为原有基于 FCD 浮动车数据采集的路况信息的有效补 充。

\section{2. 交通视频实时路况分析方法总体流程}

人眼可以很容易地通过交通实时视频区别出路段是否 拥堵。通过对人眼识别道路拥堵的过程进行分析, 我们发 现主要是由于拥堵路段车辆分布具有以下特点: 一、拥堵 路道车辆较多; 二、拥堵路道车辆间距较小。本文基于拥 堵路段的上述特点, 对交通视频图像进行处理, 并提出一 种基于视觉空间转换的网格化方法, 实现道路特征提取与 实时路况分析。具体处理流程如下: 确定道路区域, 将交 通视频原始彩色图像做灰度化处理及平滑操作, 然后在道 路区域中采用基于背景差分法进行运动物体提取, 得到二 值化图像; 通过对道路区域进行视觉空间网格化, 并根据 上述拥堵路段车辆分布特点, 对二值化图像进行分析, 计 算路况特征指数与道路拥堵指数, 以判断路段是否拥堵。

\section{3. 图像预处理}

\section{1 确定道路区域}

为消除道路以外的物体对路况分析的影响, 在进行图 像处理之前, 需根据交通视频探头的位置与朝向, 采用预 先人工设定的方法沿道路边缘设置兴趣道路区域 ${ }^{[3]}$ 。为便 于后续分析处理, 将兴趣道路区域设置为四边形。

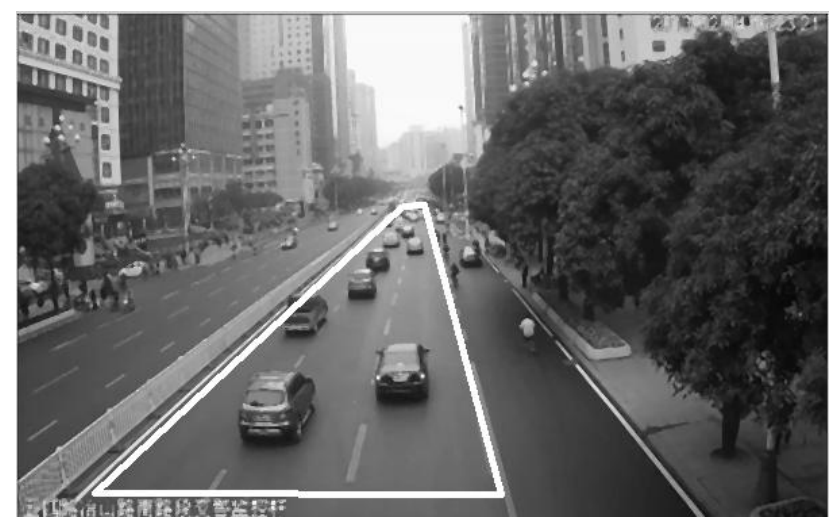

图 1 在视频图像上设置兴趣道路区域

\section{2 灰度化处理}

采用的是加权平均值法将彩色图像中的每个像素点都 转换成 8 bit 长度的亮度值, 使所有像素的灰度值都在 $[0$, 255] 范围之内 [4]。

\section{3 平滑处理}

采用了中值滤波对已经进行了灰度化的图像进行平滑 操作, 去除视频图像中的噪声, 并保留原图像中的轮廓信 息 ${ }^{[5]}$ 。

\section{4 背景差分二值化}

背景差分法首先是要构建视频场景的背景图像, 然后将把 当前帧图像与背景图像的数据进行相减, 也就是说做差值, 再进行阈值二值化。这样就得到了将运动目标从固定背景 中分割出来的二值化图像的数据。如图 3 白色部分为从图 2 中提取出的运动目标图像。

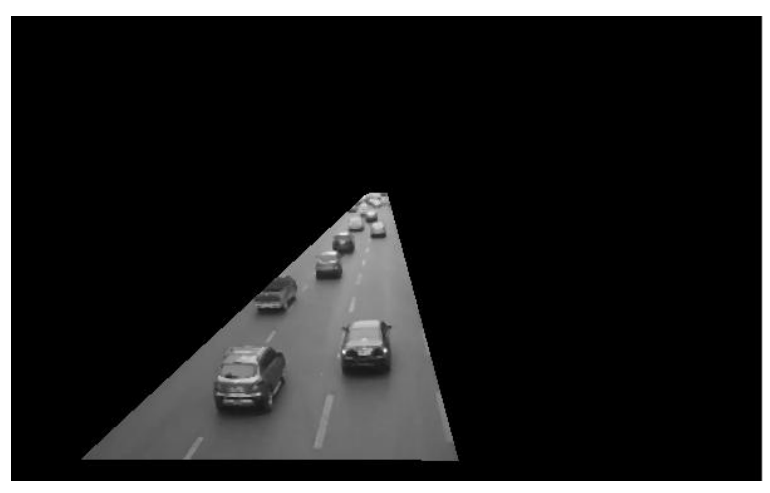

图 2 道路当前帧图像



图 3 运动目标二值化图

\section{5 运动目标填充}

由于车身存在部分较大的噪声点, 运动目标二值化后 的图像往往存在黑色空隙。因此, 可对车辆内部黑色部分 进行填充处理。本文通过判定黑像索点周边白像索的分布 情况, 来确定是否将该黑像索点置为白像索点。对一个黑 像索点 $(\mathrm{x}, \mathrm{y})$, 其周边共有 $(\mathrm{x}-1, \mathrm{y}-1),(\mathrm{x}, \mathrm{y}-1),(\mathrm{x}+1, \mathrm{y}-1)$, $(\mathrm{x}+1, \mathrm{y}),(\mathrm{x}+1, \mathrm{y}+1),(\mathrm{x}, \mathrm{y}+1),(\mathrm{x}-1, \mathrm{y}+1),(\mathrm{x}-1, \mathrm{y})$ 八个像索点, 若该黑像索点周边的八个像索点中有三个及以上像索点均 为白像索点, 则将该黑像索点置为白色。通过该方法, 可 
将车辆内部大部分黑色区域置为白色, 为后续路况分析提 供更精确的基础数据。图 4 为经填充后的运动目标。

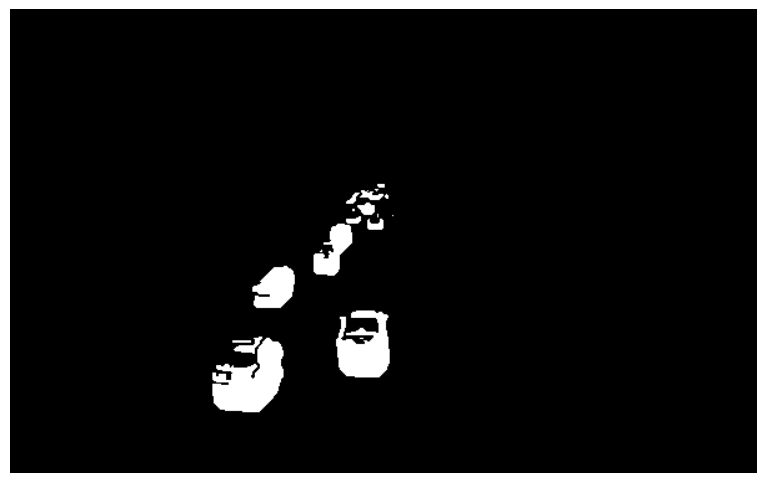

图 4 填充运动目标

\section{4. 实时路况分析}

\section{1 视觉空间转换的兴趣道路网格化}

在图像预处理环节, 我们已将兴趣道路区域设置为四 边形。在本环节, 本文提出了一种基于视觉空间转换的网 格化方法, 对四边形道路区域进行处理, 将该区域划分为 多个小四边形。

视觉空间转换网格化分为纵向网格化与横向网格化两 步。纵向网格化是沿车道线方向将道路进行 $\mathrm{m}$ 等分, 然后 连接两边各等分点。而横向网格化则相对复杂。交通视频 图像是三维空间在二维空间上的投影, 人眼可通过经验判 别视频中道路的远端与近端, 而计算机处理时需将二维图 像中的道路长度转换为三维空间道路长度后, 才能反应出 车辆在道路中的实际分布。因此, 在进行横向网格化时, 需对兴趣区域四边形中与道路走向平行的两条边进行视觉 空间转换。

根据图 5 所示, $\mathrm{h}$ 为摄像头高度, 1 为可视路段总长度, $\mathrm{k}$ 为可视路段起始点, 设将路段等分为 $\mathrm{n}$ 段, 则每段长度 为 $1 / n$ 。 $\theta i(i \in[1, n])$ 为摄像头到第 $i-1$ 等分点连线与第 $i$ 个等 分点连线的夹角, $\theta$ 为可视路段总夹角。

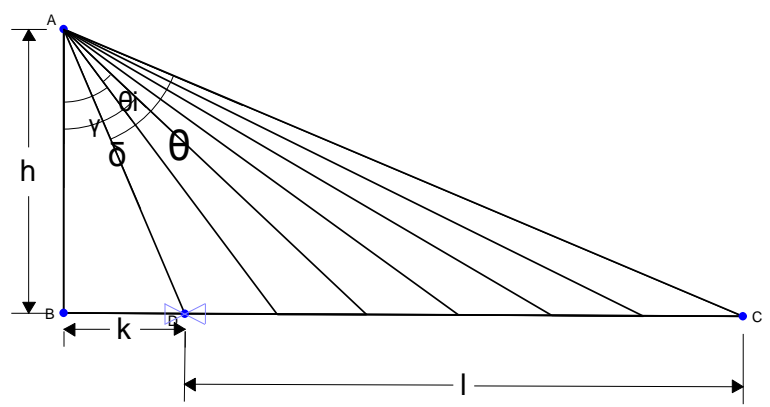

图 5 横向网格化

$$
\begin{aligned}
\tan \theta i & =\tan (\delta-\gamma)=\frac{\tan \gamma-\tan \gamma}{1+\tan \delta * \tan \gamma} \\
& =\frac{(l / n * i+k) / h-(l / n *(i-1)+k) / h}{1+((l / n * i+k) / h) *((l / n *(i-1)+k) / h)} \\
& =\frac{h^{*} l / n}{h^{2}+(l / n * i+k)^{2}-(l / n * i+k) * l / n} \\
\theta i & =\arctan \frac{h * l / n}{h^{2}+(l / n * i+k)^{2}-(l / n * i+k) * l / n}
\end{aligned}
$$

$$
\theta i=\arctan \frac{l * h}{h^{2}+(l+k) * k}
$$

对于同一个交通视频图像, 摄像头高度 $\mathrm{h}$ 为常数, 可 视道路长度 1 及可视路段起始点 $\mathrm{k}$ 均可实地测量, $\mathrm{n}$ 为参数, 因此, 可以通过公式 2 计算出夹角 $\theta i$, 本文以此夹角与总 夹角的比值作为横向网格化的划分比例。

假设 $\mathrm{m}=10, \mathrm{n}=20$, 则网格化结果如图 6 所示。

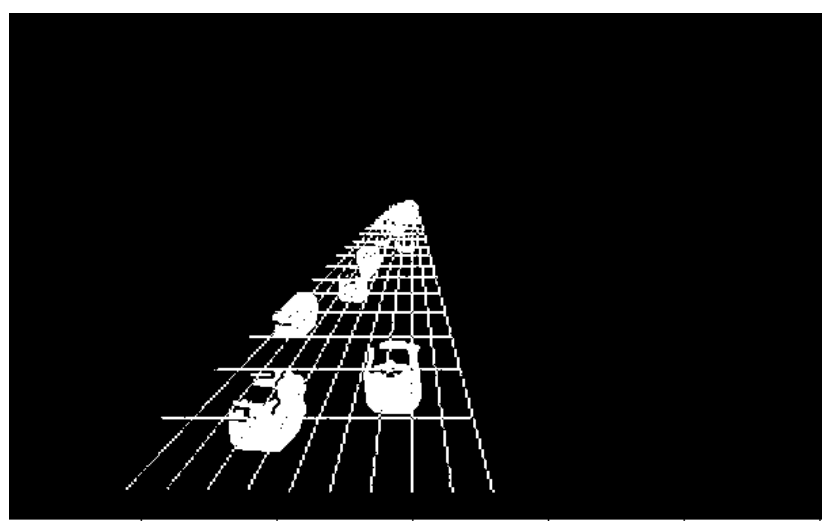

图 6 道路区域网格化

为进一步减少噪声点的影响, 可将某网格中白像索 占总像索点的比值与指定阈值相比较, 如比值大于阈值, 则可将该网格认为是有车网格; 若比值小于阈值, 将该网 格认为是无车网格。

\section{2 路况特征指数}

通过图像预处理, 原始交通视频图片已转换为兴趣路 段的车辆分布二值化图像。基于二值化图像, 并结合兴趣 道路网格, 可进行路况分析。根据拥堵路段车辆分布特点, 本文定义了以下两个路况特征指数用于衡量道路车辆拥堵 情况: 


\section{(1)车辆占比指数}

车辆多是道路拥堵最主要的原因, 车辆占比指数用于 衡量指定道路车辆的多少。定义车辆占比指数为有车网格 与总网格的比值, 设 $w$ 为有车网格的数量, $m * n$ 为总网格数 量, $r 1$ 为车辆占比指数, 即 $r 1=w /(m * n) 。 r 1$ 越大, 该路段 车辆越多, 道路拥堵可能性越大; $r 1$ 越小, 该路段车辆越 少，道路拥堵可能性越小。

\section{(2)车辆间距指数}

为保证行车安全, 车辆在快速行驶时会保持一定车距, 拥堵道路具有车辆间距较窄的特征。车辆间距指数 $\mathrm{r} 2$ 用于 衡量指定道路车辆间距的大小。依次对纵向网格（沿道路 方向) 进行处理, 设第 $\mathrm{i}$ 列纵向网格中最大连通无车网格 数量为 $B i, \sum_{i=1}^{m} B i / m$ 为各列平均最大连通无车网格数 量, $\mathrm{r} 2$ 为车辆间距指数, 则 $\mathrm{r} 2=\frac{\sum_{i=1}^{m} B i / m}{n}$ 。r2 越大, 该 路段车辆间距越大, 道路拥堵可能性越小; $r 2$ 越小, 该路 段车辆间距越小, 道路拥堵可能性越大。

\section{3 道路拥堵指数}

基于上述路况特征指数, 本文定义道路拥堵指数 $\mathrm{r}$ 用 于评估道路的拥堵程度。道路拥堵指数 $\mathrm{r}$ 是对路况特征指 数进行加权平均, 具体为 $r=r 1 * A+(1-r 2) * B \quad(A+B=1)$ 。考 虑到车辆数量是评估道路拥堵的最重要的因素, 在实际应 用中, 我们将 $\mathrm{r} 1$ 对应的权重提高。道路拥堵指数 $\mathrm{r}$ 的取值 范围介于 0 与 1 之间。当 $\mathrm{r}$ 值越大, 道路越拥堵。通过设 置经验区间, 可根据 $\mathrm{r}$ 值将道路实时路况等级确定为顺畅、 缓慢和拥堵。

\section{5. 实验结果及结论}

实验样本选取同一路段不同时段的 100 张道路图像。 仿真测试环境为: $2.53 \mathrm{GHz} \mathrm{CPU}, 2 \mathrm{G}$ 内存, 操作系统为 Windows7, 在 MATLAB7. 0 平台上编程实现。算法先对图像 执行确定道路区域、灰度化处理、平滑处理、背景差分二 值化、运动目标填充等的预处理环节, 并对道路区域进行 网格化, 然后计算道路图像的路况特征指数, 并通过加权 平均后转换为路况识别指数。每张图像平均处理时间约为 1.2 秒, 与实际道路图像对比, 实验正确检测率约为 $91 \%$ 。以 下为部分实验结果:
表 1 部分实验结果

\begin{tabular}{|c|c|c|c|c|}
\hline 道路图像 & $\begin{array}{c}\text { 车辆占比指数 } \\
\text { (权重 } 70 \%)\end{array}$ & $\begin{array}{c}\text { 车辆间距指数 } \\
\text { (权重 } 30 \%)\end{array}$ & $\begin{array}{c}\text { 道路拥 } \\
\text { 堵指数 }\end{array}$ & 识别结果 \\
\hline & 0.37 & 0.355 & 0.4525 & 顺畅 \\
\hline & 0.115 & 0.475 & 0.238 & 顺畅 \\
\hline & & & & \\
\hline & 0.585 & 0.16 & 0.6615 & 缓慢 \\
\hline
\end{tabular}

实验结果表明本文提出的结合视觉空间转换的交通视 频路况分析方法可有效识别道路拥堵情况, 算法检测准确 率高, 识别速度快, 满足实际应用中对准确性与及时性的 要求。但在夜晚环境及不规则道路处理上还存在局限性, 这也是下一步要完成的工作。

\section{参考文献(References)}

[1] Yang Yonghui, HUANG Lei, LIU Changping, "Measurement of vehicle queue length based on video analysis," Application Research of Computers, vol. 28, no. 2, pp.1037-1041, 2011.

[2] ZHOU Tao, ZHANG Jiye, "Video images vehicle detection and recognition," Computer Engineering and Application, vol.47, no.19, pp.166-169,2011

[3] Wei Wu, Gong Shufeng, Gong Shuchao, "Improved Video based vehicle detection and Identification Method," Computer Measurement and Control, vol.18, no.1, pp.20-22,2010

[4] FENG Yun-peng, Zhang Na, MA Rong, "Research and Implementation of Video based Vehicle Finding System," Computer Simulation, vol.29, no.2, pp.367-371,2012

[5] XU Zheng, WANG Yichao, HUANG Xuan, LUO Hua, XIONG Hui, "License Plate Recognition and Speed Based on the Traffic Camera Video," JIANGXI SCIENCE, vol.30, no.4, pp.528-531,2012 